\title{
In vivo functional analysis of L-rhamnose metabolic pathway in Aspergillus niger: a tool to identify the potential inducer of RhaR
}

Claire Khosravi, Roland Sándor Kun, Jaap Visser, María Victoria Aguilar-Pontes, Ronald P. de Vries (D) and Evy Battaglia

\begin{abstract}
Background: The genes of the non-phosphorylative L-rhamnose catabolic pathway have been identified for several yeast species. In Schefferomyces stipitis, all L-rhamnose pathway genes are organized in a cluster, which is conserved in Aspergillus niger, except for the Ira-4 ortholog (IraD). The A. niger cluster also contains the gene encoding the L-rhamnose responsive transcription factor (RhaR) that has been shown to control the expression of genes involved in L-rhamnose release and catabolism.

Result: In this paper, we confirmed the function of the first three putative L-rhamnose utilisation genes from $A$. niger through gene deletion. We explored the identity of the inducer of the pathway regulator (RhaR) through expression analysis of the deletion mutants grown in transfer experiments to L-rhamnose and L-rhamnonate. Reduced expression of L-rhamnose-induced genes on L-rhamnose in IraA and IraB deletion strains, but not on L-rhamnonate (the product of LraB), demonstrate that the inducer of the pathway is of L-rhamnonate or a compound downstream of it. Reduced expression of these genes in the IraC deletion strain on L-rhamnonate show that it is in fact a downstream product of L-rhamnonate.
\end{abstract}

Conclusion: This work showed that the inducer of RhaR is beyond L-rhamnonate dehydratase (LraC) and is likely to be the 2-keto-3-L-deoxyrhamnonate.

Keywords: L- rhamnose catabolic pathway, Inducer, Transcriptomics, Pectinase, Gene regulation

\section{Background}

Plant biomass is mainly composed of polysaccharides. In nature, fungi secrete a broad range of polysaccharide degrading enzymes to release monosaccharides, which are then used as nutrients. Pectin is a complex plant cell wall polysaccharide that can be divided into four substructures: homogalacturonan (HGA), xylogalacturonan (XGA) and rhamnogalacturonan I and II (RG-I and RGII) [1]. The backbone of RG-I is composed of alternating L-rhamnose and D-galacturonic acid residues. Long side chains of L-arabinose (arabinan), D-galactose (galactan),

\footnotetext{
* Correspondence: r.devries@westerdijkinstitute.nl

Fungal Physiology, Westerdijk Fungal Biodiversity Institute \& Fungal

Molecular Physiology, Utrecht University, Uppsalalaan 8, 3584, CT, Utrecht, The Netherlands
}

(c) The Author(s). 2017 Open Access This article is distributed under the terms of the Creative Commons Attribution 4.0 International License (http://creativecommons.org/licenses/by/4.0/), which permits unrestricted use, distribution, and reproduction in any medium, provided you give appropriate credit to the original author(s) and the source, provide a link to the Creative Commons license, and indicate if changes were made. The Creative Commons Public Domain Dedication waiver (http://creativecommons.org/publicdomain/zero/1.0/) applies to the data made available in this article, unless otherwise stated.

or a mixture of L-arabinose and D-galactose residues (arabinogalactan) can be attached to these L-rhamnose residues $[2,3]$.

$A$. niger is able to efficiently degrade pectin and can utilize L-rhamnose also as the sole carbon source. $A$. niger possesses enzymes that are able to enzymatically release L-rhamnose from RG-I. Endo- and exorhamnogalacturonase, $\alpha$-rhamnosidase, rhamnogalacturonan lyase and rhamnogalacturonan acetyl esterase are all active towards the main chain of RG-I $[4,5]$.

An L-rhamnose transporter (RhtA) has been characterized recently in $A$. niger [6]. Interestingly, it has been found that $r h t A$ is co-localized with the $\alpha-\mathrm{L}$ rhamnosidase gene $r h a B$. The transcriptional profile of $r h t A$ and $r h a B$ genes during a time-course growth 
experiment of $A$. niger on L-rhamnose suggests not only that there is a coordinated role in the release and the transport of L-rhamnose during consumption but also that strong activation of these two genes do not require high concentrations of L-rhamnose but only low levels [6].

Two pathways are known for the catabolism of Lrhamnose: a phosphorylative and a non-phosphorylative pathway. The phosphorylative pathway is only found in bacteria such as Escherichia coli [7], while the nonphosphorylative pathway is found in bacteria and yeasts [8-12]. In the non-phosphorylative pathway of Schefferomyces stipitis, L-rhamnose is converted to pyruvate and L-lactaldehyde via four metabolic reactions involving a L-rhamnose-1-dehydrogenase (RHA1) that oxidizes L-rhamnose to L-rhamnono-Y-lactone, a L-rhamnono- $\gamma$ lactonase (LRA2) that converts L-rhamnono- $\gamma$-lactone to L-rhamnonate, a L-rhamnonate dehydratase (LRA3) that converts L-rhamnonate to 2-keto-3-deoxy-L-rhamnonate (L-KDR) and a 2-keto-3-deoxy-L-rhamnonate (L-KDR) aldolase (LRA4) that converts the 2-keto-3-deoxy intermediate to pyruvate and L-lactaldehyde (Fig. 1a). All the genes (RHA1, LRA2, LRA3 and LRA4) have been identified in S. stipitis and the corresponding enzymes have been biochemically characterized $[8,13,14]$. The RHA1, LRA2, LRA3, LRA4 genes are organized in a cluster in S. stipitis together with a transcription factor with a Zn (II)2Cys6 zinc binuclear cluster domain TRC1 (Fig. 1c). This gene cluster is either fully or partially characterized in other fungal species [13]. In A. niger, the homologous genes of RHA1, LRA2, LRA3 designated as $\operatorname{lraA}$, $\operatorname{lraB}$ and $\operatorname{lraC}$, as well as the L-rhamnose regulator RhaR [15] are organized in a cluster (Fig. 1c). However, no LRA4 homolog has been found in the cluster in the A. niger genome (Fig. 1b). Only one of the $A$. niger L-rhamnose metabolic genes, $\operatorname{lraC}$, has been biochemically characterized to encode a L-rhamnonate dehydratase by expressing it in Saccharomyces cerevisiae [16]. A double deletion of $\operatorname{lraC}$ and $\operatorname{lra} A$ in $A$. niger resulted in a strain that exhibited no L-rhamnose dehydrogenase activity and could not consume or grow on L-rhamnose [17]. It remains unknown which gene function, $\operatorname{lraA}$ or $\operatorname{lraC}$, is actually the cause of the inability of $A$. niger to grow on L-rhamnose. As the in vivo function of none of the three individual L-rhamnose pathway genes (lraA, $\operatorname{lraB}$ and $\operatorname{lraC}$ ) has been studied yet in $A$. niger our first goal was to perform an in vivo analysis of their function by making single gene deletion mutants in $A$. niger. Secondly, we searched and selected lraD candidate genes in the $A$. niger genome for making the corresponding gene deletions. And thirdly we made use of the metabolic mutants to identify the inducer of

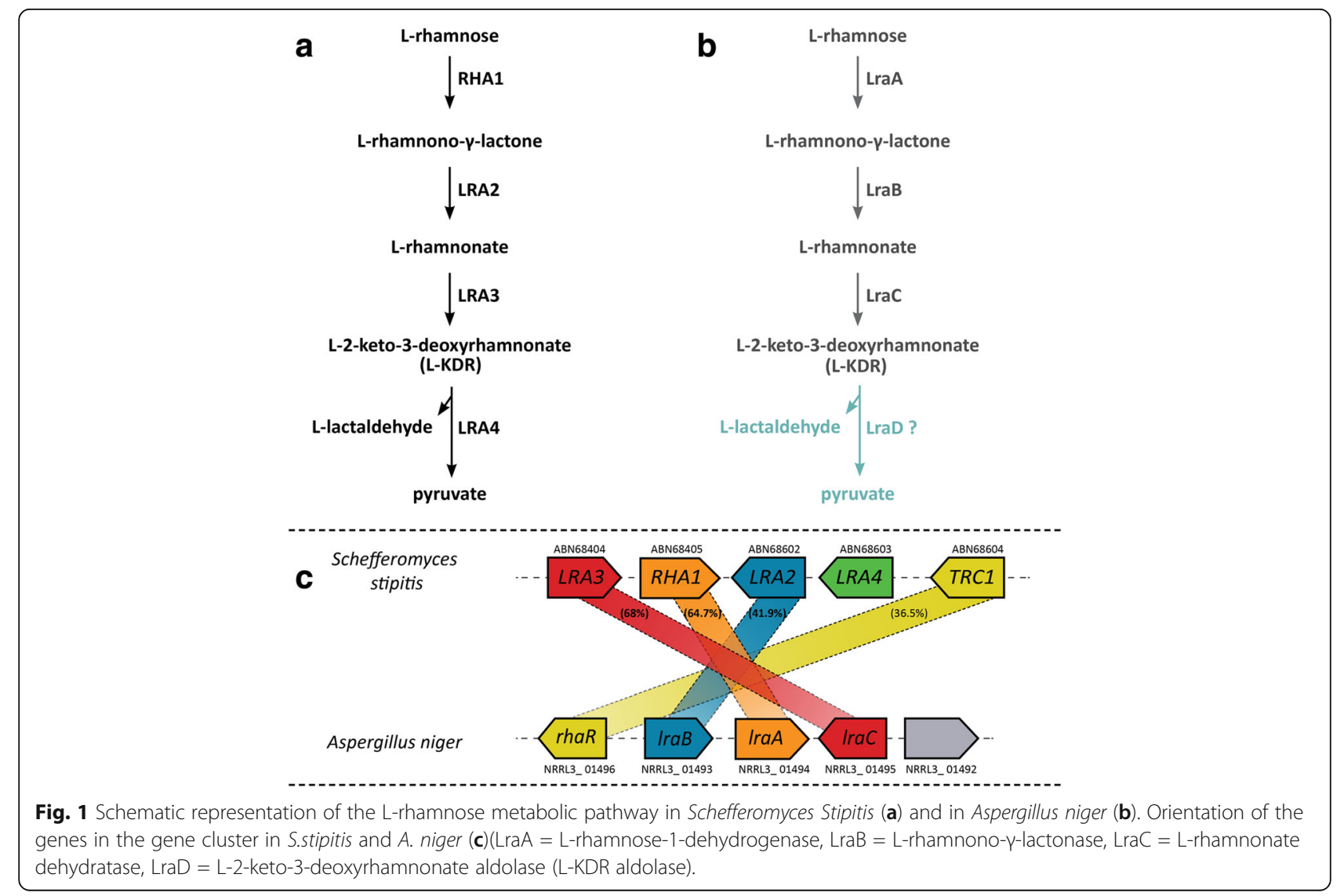


RhaR in A. niger. In A. niger, RhaR has been shown to control expression of genes involved in RG-I degradation, the L-rhamnose transporter gene $(r h t A)$ as well as the L-rhamnose catabolic genes ( $\operatorname{lr} a A, \operatorname{lra} B$ and $\operatorname{lra} C$ ) during growth on L-rhamnose $[6,15,18]$. It has been suggested that L-rhamnose or a conversion product thereof could be the inducer of RhaR [15]. Blocking the individual steps in the L-rhamnose pathway would thus allow the identification of the inducer. Either the sugar itself or a metabolic pathway intermediate has been shown to induce other plant biomass related transcriptional regulators, such as L-arabinose/L-arabitol for AraR [19], D-xylose for XlnR [20] and 2-keto-3-deoxy-L-galactonate for GaaR-GaaX activator-repressor module [21]. Therefore, we did not make the deletion mutant of $r h t A$ or any other putative L-rhamnose transporter in order to allow always Lrhamnose uptake.

Gene deletion and transcriptomic analysis performed in this study showed that $\operatorname{lraA}, \operatorname{lraB}$ and $\operatorname{lraC}$ are required for growth on L-rhamnose and confirmed that RhaR plays an important role in the regulation of L-rhamnose catabolism and the degradation of pectin. The results also indicate that L-rhamnose, L-rhamnonoY-lactone and L-rhamnonate are not the inducers of RhaR, but that this is a compound located further down in the metabolic pathway.

\section{Methods}

\section{Strains, media and culture conditions}

A. niger strain CBS 141257 (Table S1) was used to create the $\Delta \operatorname{lraA}, \Delta \operatorname{lraB}$ and $\Delta \operatorname{lraC}$ strains. The CBS 141257 strain was obtained by transformation of N593.20 with uridine (pyrG) from Aspergillus oryzae [22]. All A. niger strains were grown at $30{ }^{\circ} \mathrm{C}$ using minimal medium (MM, pH 6) or complete medium (CM, pH 6) [23] with $1.5 \%$ of agar. Spores plates contained CM with $2 \%$ D-glucose. Plates used in the growth profile contained $\mathrm{MM}+25 \mathrm{mM}$ monosaccharides, inoculated with 1000 spores.

Liquid cultures of two biological duplicates were inoculated with $10^{6}$ spores $/ \mathrm{ml}$ and incubated in a rotary shaker at $250 \mathrm{rpm}$ and $30{ }^{\circ} \mathrm{C}$. Pre-cultures for RNA isolation were grown for $16 \mathrm{~h}$ in $1 \mathrm{~L}$ Erlenmeyer flasks containing $250 \mathrm{ml} \mathrm{CM}$ with $2 \%$ D-fructose. Mycelium was washed with MM and transferred for $2 \mathrm{~h}$ in $250 \mathrm{ml}$ Erlenmeyer flasks containing $50 \mathrm{ml}$ MM supplemented with $25 \mathrm{mM}$ L-rhamnose for RNA-seq. For qPCR analysis, mycelium was transferred for $2 \mathrm{~h}$ in $50 \mathrm{ml}$ Erlenmeyer flaks containing $10 \mathrm{ml} \mathrm{MM}$ supplemented with $25 \mathrm{mM}$ carbon source L-rhamnose (Fluka) or L-rhamnonate (Sigma). Mycelium was harvested by vacuum filtration, dried between tissue paper and frozen in liquid nitrogen. New strains were deposited at the Westerdijk Fungal
Biodiversity Institute with strain numbers indicated in Additional file 1: Table S1.

\section{Molecular biology methods and fungal transformation}

To construct the deletion cassettes, the upstream and downstream flanks of the genes ( $\operatorname{raA}, \operatorname{lraB}$ and $\operatorname{lraC}$ ) were amplified using PCR with gene specific primers (Additional file 2: Table S2). The upstream flank reverse primer and downstream flank forward primer carried homologous sequences, overlapping the ends of the pyrG selection cassette. The pyrG cassette was amplified from pRV1005 and purified using the Wizard_SV Gel and PCR Clean-Up Start-Up Kits (Promega). These three fragments were combined in a fusion PCR reaction, to generate the deletion cassette. The fusion PCR mixture contained $1 \mu \mathrm{l}$ of each PCR product, $12.5 \mu \mathrm{L}_{\text {GoTaq }}$ Long PCR Master Mix (Promega), $2 \mu \mathrm{L}$ of each $10 \mathrm{mM}$ primer in a total volume of $25 \mu \mathrm{L}$. The following PCR conditions were used: $2 \mathrm{~min}$ at $94{ }^{\circ} \mathrm{C}, 20 \mathrm{~s}$ at $94{ }^{\circ} \mathrm{C}, 20 \mathrm{~s}$ at $58{ }^{\circ} \mathrm{C}, 5 \mathrm{~min}$ at $68{ }^{\circ} \mathrm{C}$ for 30 cycles and finally an additional $10 \mathrm{~min}$ at $68^{\circ} \mathrm{C}$. The amplified deletion cassettes were purified using the Wizard_SV Gel and PCR CleanUp Start-Up Kits (Promega). Purified DNA $(5 \mu \mathrm{g})$ of the deletion cassette was used to transform $A$. niger. Protoplast-mediated transformations of $A$. niger were performed as described [24]. DNA was isolated from frozen mycelium, ground with a Tissue Lyser (QIAGEN) using a standard chloroform/phenol extraction. For screening of the A. niger transformants, a PCR was performed using genomic DNA of the transformants. Gene-specific sets of primers were used to check the absence or presence of the ORF (Additional file 2: Table S2). The resulting strains are listed in Additional file 1: Table S1.

\section{RNA extraction, CDNA library preparation and RNA-seq}

Total RNA was extracted from mycelium ground in a Tissue Lyser (QIAGEN) using the TRIzol reagent (Invitrogen, Breda, The Netherlands) according to the instructions of the manufacturer. Total RNA samples were purified with the NucleoSpin RNA Clean-up Kit (Macherey-Nagel). Contaminating gDNA was removed by an rDNase solution directly on the silica membrane. RNA integrity and quantity were analyzed on a $1 \%$ agarose gel using gel electrophoresis and with the RNA6000 Nano Assay using the Agilent 2100 Bioanalyzer (Agilent Technologies). cDNA library preparation and sequencing reactions were conducted at BGI Tech Solutions Co., Ltd. (Hong Kong). Illumina library preparation, clustering, and sequencing reagents were used throughout the process following the manufacturer's recommendations (http://illumina.com). mRNA was purified using poly- $T$ oligonucleotide-attached magnetic beads and then fragmented. The first and second strands cDNA were synthesized and end repaired. Adaptors were 
ligated after adenylation at the 3 ' end. After gel purification, cDNA templates were enriched by PCR. cDNA libraries were validated using the Agilent 2100 Bioanalyzer (Agilent Technologies) and quantified by qPCR. Singleread samples were sequenced using Illumina $\mathrm{HiSeq}^{\text {тm }} 2000$ platform (http://illumina.com). On average 51 bp sequenced reads were constituted, producing approximately a yield of $360 \mathrm{MB}$ raw sequence for each sample.

\section{RNA-seq data analysis and functional annotation}

Raw reads were produced from the original image data by base calling. After data filtering, the adaptor sequences, highly ' $\mathrm{N}$ ' containing reads ( $>10 \%$ of unknown bases) and low quality reads (more than $50 \%$ bases with quality value of $<5 \%$ ) were removed. After data filtering, on average, 99\% clean reads remained in each sample. Clean reads were then mapped to the genome of $A$. niger NRRL3 (http://genome.jgi.doe.gov/ Aspni_NRRL3_1/Aspni_NRRL3_1.home.html) using BWA [25, 26]. No more than two mismatches were allowed in the alignment. On average, 70\% total mapped reads to the gene was achieved. We refer to $A$. niger gene IDs based on the most up-to-date and accurate annotation of the $A$. niger NRRL3 genome (http://genome.fungalgenomics.ca/). The gene expression level (FPKM) was calculated by using RSEM tool [27]. Genes with expression value higher than 120 were considered highly expressed (approximately top 5\%) and differential expression was identified by Student's t-test with a $P$-value cutoff 0.05 . The RNA-seq data have been submitted to Gene Expression Omnibus (GEO) [28] with accession number: GSE99865.

\section{qRT-PCR analysis}

cDNA was prepared from total RNA $(2.5 \mu \mathrm{g})$ using Thermoscript RT (Invitrogen) according to the instructions of the manufacturer. The sequences of all primers for qRT-PCR analysis were designed using the Primer Express 3.0 software (Applied Biosystems). The primers were tested to determine the optimal primer concentrations and efficiency. Combinations of the $50 \mathrm{nM}, 300 \mathrm{nM}$ and $900 \mathrm{nM}$ (final concentration) per primer pair were checked, and based on the dissociation curve the optimal primer concentration per primer pair was chosen. The primer sequences of the tested genes and the reference gene are listed in Additional file 3: Table S3. qPCR analysis was performed by using the ABI 7500 fast real-time PCR system (Applied Biosystems). The reactions consisted of $2 \mu \mathrm{l}$ forward and reverse primers at optimal concentration, 20 ng cDNA sample, $10 \mu \mathrm{l}$ ABI Fast SYBR Master Mix (Applied Biosystems), and water to a final volume of $20 \mu \mathrm{l}$. The cycling parameters were $95{ }^{\circ} \mathrm{C}$ for $20 \mathrm{~s}$, followed by 40 cycles of $95{ }^{\circ} \mathrm{C}$ for $3 \mathrm{~s}$ and $60{ }^{\circ} \mathrm{C}$ for $30 \mathrm{~s}$. A dissociation curve was generated to verify that a single product was amplified. Transcript levels were normalized against the histone $\mathrm{H} 2 \mathrm{~B}$ gene expression and quantified according to the formula 2 $-(\mathrm{Ct}$ gene $\mathrm{X}-\mathrm{Ct} \mathrm{H} 2 \mathrm{~B})$ [29]. Two biological and three technical replicates were analyzed.

\section{Results}

In vivo effects of deleting the L-rhamnose metabolic genes The candidate genes for the first three enzymatic reactions of the L-rhamnose pathway are homologs of the characterized genes of $S$. stipitis [15]. The single deletions in NRRL3_08837 ( $\triangle$ lraA), NRRL3_01493 $(\triangle$ lraB $)$ and NRRL3_10522 ( $\Delta$ lraC) resulted in strains that are unable to grow on L-rhamnose (Fig. 2) compared to the reference strain. A similar growth phenotype was observed on L-rhamnose for the $\Delta r h a R$ strain (Fig. 2). The small colonies observed for the metabolic deletion mutants on L-rhamnose is background growth since the plates looked similar to

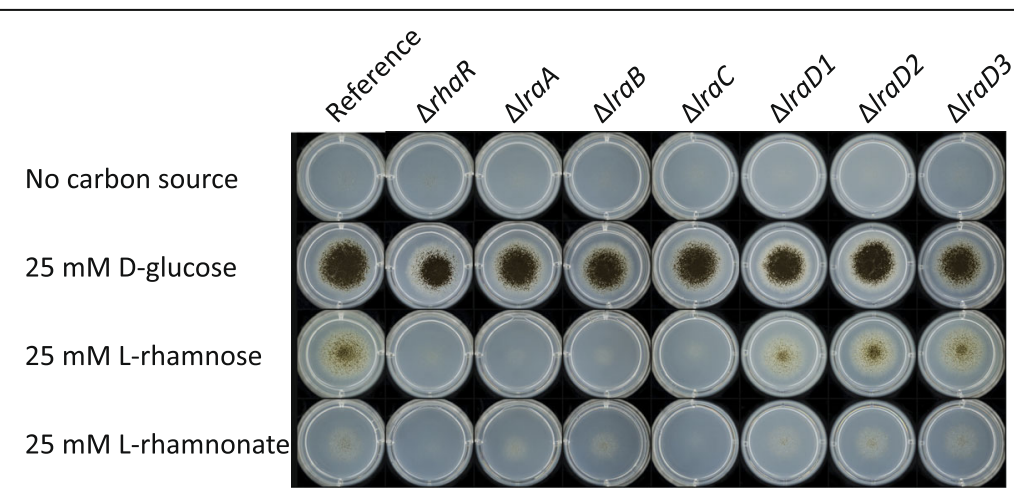

Fig. 2 Growth profile on L-rhamnose, L-rhamnonate and D-glucose of the reference strain and the deletion mutants. The reference, $\Delta /$ raA, $\Delta /$ raD1, $\Delta$ /raD2 and $\Delta /$ raD3 were grown on MM with $25 \mathrm{mM} \mathrm{L-rhamnose,} 25 \mathrm{mM} \mathrm{L-rhamnonate} \mathrm{and} 25 \mathrm{mM}$ D-glucose for 4 days at $30^{\circ} \mathrm{C}$. Spore inoculations were done with 1000 spores 
our control agar plates without any carbon source added (Fig. 2). Growth of the metabolic deletion mutants on D-glucose was similar to the reference (Fig. 2). This result indicates that all three genes of the pathway are essential for the conversion of Lrhamnose. Growth of $\triangle l r a A$ and $\Delta \operatorname{lra} B$ was reduced on L-rhamnonate, while no growth was observed in the $\Delta r h a R$ and $\Delta l r a C$ on L-rhamnonate (Fig. 2).

Previously, it was already shown by performing a bidirectional BLAST analysis that the closest homolog of $S$. stipitis LRA4 is NRRL_08779 [15]. However, this gene is not found in the $A$. niger L-rhamnose cluster (Fig. 1c), it is not induced on L-rhamnose compared to D-glucose and its expression is not affected in the $\Delta r h a R$ mutant on L-rhamnose compared to the reference strain (Additional file 4: Table S4). In addition, it has a very low expression level in all growth conditions tested. In order to search for candidate genes for the L-KDR aldolase ( $\operatorname{raD})$, we analyzed the $A$. niger genome for genes containing any of the InterPro and PFAM domains (Additional file 4: Table S4), similar domains to those found in S. stipitis LRA4 [13]. The most similar gene to S. stipitis LRA4, NRRL_08779, is not significantly expressed on L-rhamnose in the micro-array data, nor in any other in-house micro-array or RNAseq datasets, and was also not detected by qPCR on either L-rhamnose or L-rhamnonate (data not shown), and was therefore excluded as a candidate for this enzymatic function in $A$. niger. We therefore compared the expression between L-rhamnose and D-glucose of all candidate genes containing the relevant domains and ranked them by foldchange between L-rhamnose and D-glucose, resulting in the selection of three candidate genes (NRRL3_03899, NRRL3_05649, NRRL3_06731). The first candidate was 39-fold up-regulated, the second one was 24-fold up-regulated and the third one was 3-fold up regulated on Lrhamnose in comparison to D-glucose (Additional file 4: Table S4). The other genes were either not induced on L-rhamnose compared to D-glucose or not expressed at all. These three candidate genes for $\operatorname{lraD}$ were deleted in the A. niger CBS 141257. Growth phenotypic analysis of the mutants ( $\Delta$ lraD1, $\Delta$ lraD2, $\Delta$ lraD3) was performed and growth on L-rhamnose was compared to the reference strain. Deletion of the genes did not affect growth on L-rhamnose and L-rhamnonate (Fig. 2), suggesting that these genes are not the functional homologs of S. stipitis LRA4.

\section{L-KDR is most likely the inducer of the pathway specific regulator RhaR}

RhaR was previously identified as an L-rhamnoseresponsive transcription factor gene with a role in induction of genes involved in L-rhamnose transport, catabolism and in RG-I degradation $[6,15]$. To test the effect of the three metabolic gene deletions on the induction of L-rhamnose responsive genes, RNA-seq analysis was performed. The reference strain and the $\mathrm{KO}$ strains $\triangle \operatorname{lra} A, \Delta \operatorname{lraB}, \Delta \operatorname{lra} C$ and $\triangle r h a R$ were pre-grown in liquid cultures containing $M M$ with fructose and then transferred to MM with L-rhamnose. RNA-seq analysis showed that the expression levels of $\operatorname{lraA}, \operatorname{lraB}$ and $\operatorname{lraC}$ were close to 0 , in $\triangle \mathrm{lra} A, \triangle \mathrm{lra} B$ and $\triangle \mathrm{lraC}$, respectively (Fig. 3) confirming the deletion of these genes. When IraA, IraB or $\operatorname{lraC}$ are deleted, the expression level of the other genes of the L-rhamnose pathway is reduced (Fig. 3). A high expression level was observed for rhaR in the wild type, which was reduced to almost zero in $\Delta \operatorname{lraC}$, but only a small reduction in its expression was visible in $\triangle l r a A$ and $\triangle l r a B$ (Fig. 3). In the $\triangle r h a R$ mutant, expression of $\operatorname{lr} a A, \operatorname{lraB}$ and $\operatorname{lra} C$ is strongly reduced, confirming that these genes are under control of RhaR, as previously reported [15]. In the $\triangle \mathrm{lra} A$ and $\Delta l \mathrm{raB} \mathrm{mu}$ tant, the expression level of the L-rhamnose transporter gene $(r h t A)$ is strongly reduced (Fig. 3; Additional file 5: Table S5). However, $r h t A$ was not expressed in the $\Delta l r a C$ and $\Delta r h a R$ mutants (Fig. 3). Only one of the other candidate L-rhamnose transporter genes described previously [6] showed a similar gene expression profile in the reference strain and the metabolic mutants (NRRL3_02828).

In summary, these results show that in the $\triangle \operatorname{lraA}$, $\triangle l r a B$ or $\triangle$ lraC mutant, the transcript level of the other metabolic pathway genes and the transporter gene is reduced. It is most likely that this reduction in gene expression is due to lack of inducer formation and that accumulation of L-rhamnose, L-rhamnono-y-lactone and L-rhamnonate does not result in hyper-induction of RhaR-regulated genes as was seen previously for the $A$. niger $\triangle$ gaaC mutant during growth on $\mathrm{D}$-galacturonic acid [21]. These results also demonstrate that Lrhamnose, L-rhamnono- $\gamma$-lactone and L-rhamnonate are not the inducers of RhaR, and we therefore hypothesized that the inducer is formed further down in the Lrhamnose catabolic pathway and it therefore might be L-KDR. To strengthen this hypothesis, we checked the expression of six rhamnose-induced genes after $2 \mathrm{~h}$ of transfer of the metabolic mutants to L-rhamnonate as well. Fructose pre-grown mycelium of the reference, $\Delta r h a R, \Delta l r a A, \Delta l r a B$ and $\Delta l r a C$ strains were transferred to $M M$ supplemented with either L-rhamnose or Lrhamnonate. $\mathrm{qPCR}$ analysis showed reduced expression levels for $\operatorname{lraC}$, $\operatorname{lraB}, \operatorname{lraC}$, rhaR, rhamnogalacturonan lyase $(r g l B)$, exo-rhamnogalacturonan hydrolase $(r g x A)$ and rhamnogalacturonan acetylesterase (rgaeA) in the $\operatorname{lraA}$ and $\operatorname{lraC}$ mutants compared to the reference, after $2 \mathrm{~h}$ of culture in L-rhamnose, confirming our RNA-seq data results (Fig. 4a-g). Except for rhaR and rgaeA, the expression level of the genes is higher in $\triangle \mathrm{raB}$ compared to the reference strain (Fig. $4 \mathrm{~d}$ and g). Interestingly, all six genes were 


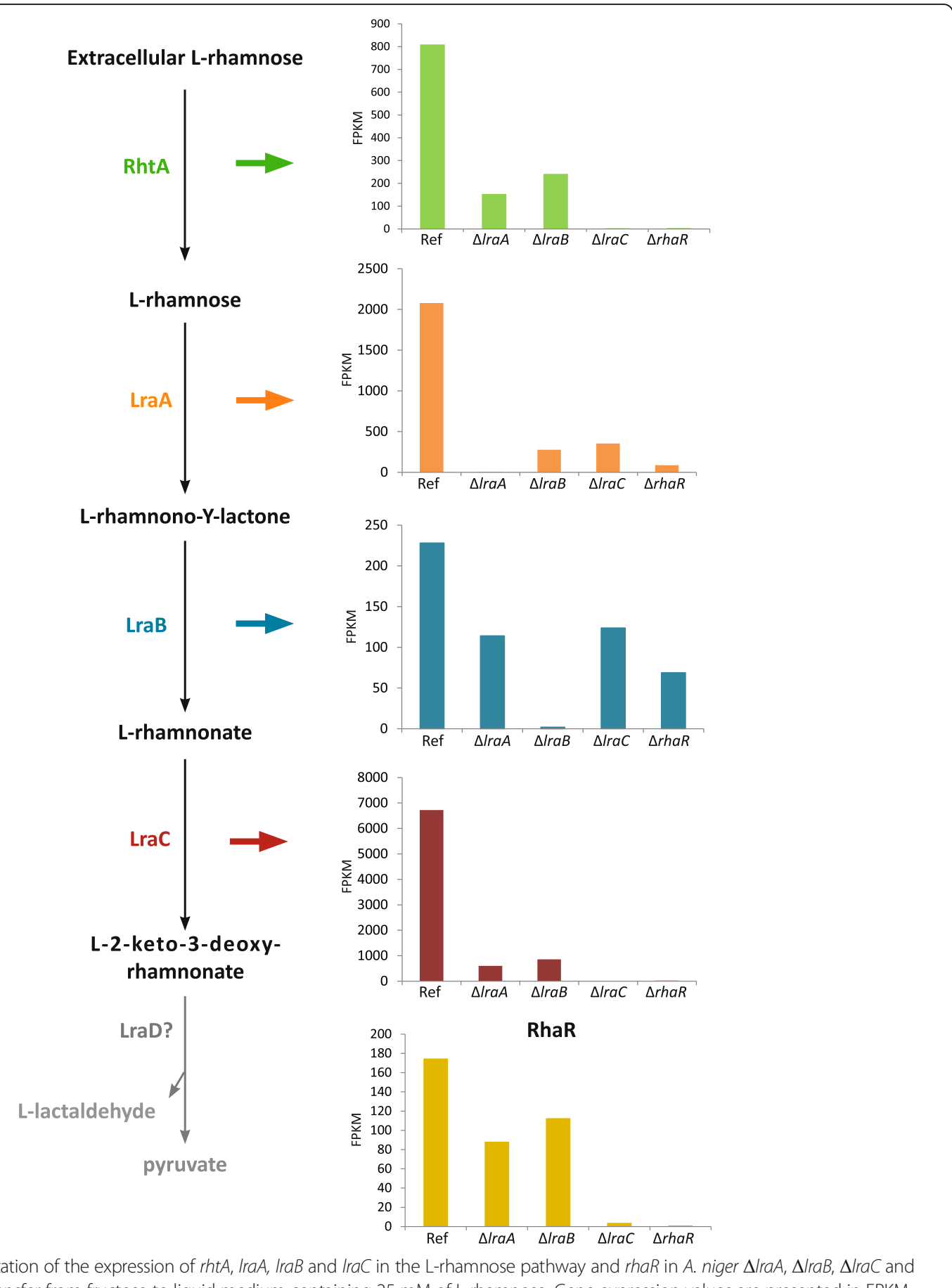

Fig. 3 Schematic representation of the expression of rhtA, IraA, IraB and IraC in the L-rhamnose pathway and rhaR in A. niger $\Delta$ rraA, $\Delta$ /raB, $\Delta$ lraC and $\Delta r h a R$ strains after $2 \mathrm{~h}$ of transfer from fructose to liquid medium containing $25 \mathrm{mM}$ of L-rhamnose. Gene expression values are presented in FPKM

induced during $2 \mathrm{~h}$ of growth of the reference strain on $\mathrm{L}$ rhamnonate (Fig. 4h-n). Transcript levels were similar to those observed for the reference strain on L-rhamnose (Fig. 4a-g). Deletion of either $\operatorname{lraA}$ or $\operatorname{lraB}$ did not affect the expression of the other metabolic genes, the rhaR gene and the three RG-I specific genes. However, strongly reduced expression levels of $\operatorname{lra} A, \operatorname{lraB}, \operatorname{rhaR}, \operatorname{rgl} B, \operatorname{rgx} A$ and $r g a e A$ were observed in the $\triangle \operatorname{lra} C$ mutant upon the
$2 \mathrm{~h}$ transfer to L-rhamnonate compared to the reference strain (Fig. 4h-n).

CAZy genes involved in degradation of the RG-I were down-regulated in the L-rhamnose pathway mutants The expression of pectinolytic genes was compared between the L-rhamnose pathway mutants and the reference strain after a transfer for $2 \mathrm{~h}$ from fructose to liquid 


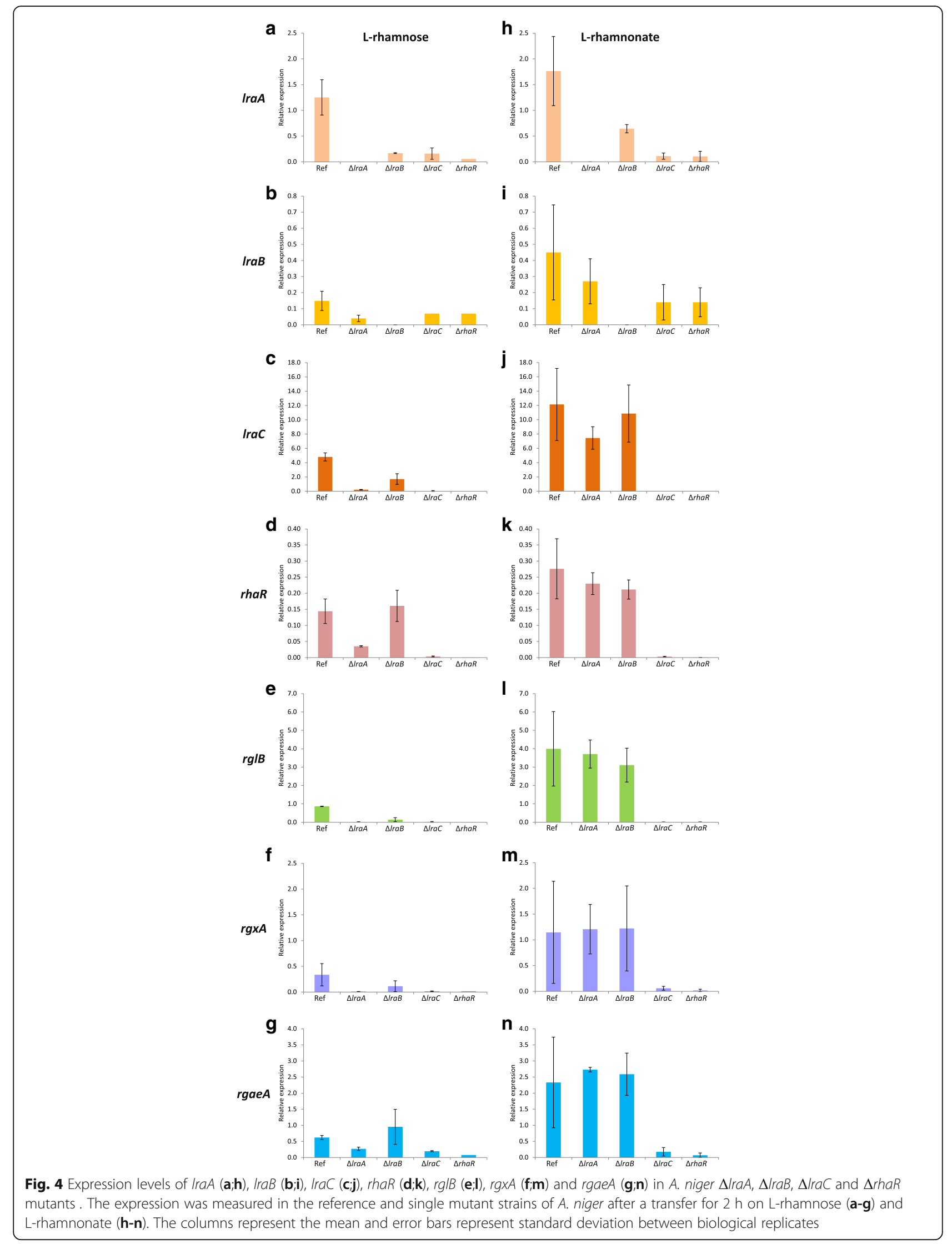


medium containing $25 \mathrm{mM}$ of L-rhamnose to study the transcript profiles of all rhamnose-induced genes in detail. Pectinolytic genes were divided into subclasses, depending on where they act on the pectin backbone: homogalacturonan (HGA), xylogalacturonan (XGA), rhamnogalacturonan-I (RG-I) and side chains (SC) (Additional file 6: Table S6). Most of the genes involved in the degradation of the RG-I backbone were down-regulated in the $\operatorname{lraA}, \operatorname{lraB}, \operatorname{lraC}$ and $r h a R$ deletion mutants compared to the reference strain: two genes encoding GH28 exo-rhamnogalacturonases ( $r g x A$ : NRRL3_02832; rgxB: NRRL3_08631), four putative GH78 $\alpha$-rhamnosidases (NRRL3_02162; NRRL3_06304; NRRL3 _03279; NRRL3_07520), one gene encoding a GH105 unsaturated rhamnogalacturonan hydrolase (urhgA; NRRL3_00839), one gene encoding a PL4 rhamnogalacturonan lyase (rglB; NRRL3_10115) and two genes encoding CE12 rhamnogalacturonan acetyl esterases (rgaeA; NRRL3_00169 and rgaeB; NRRL3_07501) (Table 1 and Additional file 6: Table S6). Two pectinolytic genes involved in the degradation of HGA and XGA, one PL1 pectin lyase (pelF; NRRL3_04153) and one CE12 pectin acetyl esterase (paeA; NRRL3_06053), showed a strongly reduced expression level on L-rhamnose in all metabolic deletion strains and in $\Delta r h a R$ compared to the reference strain (Additional file 6: Table S6). Two genes involved in the degradation of the pectin side chains, one GH3 $\beta$ xylosidase gene ( $x \ln D$; NRRL3_02451) and one GH35 $\beta-1$, 4-galactosidase gene (lacC; NRRL3_11738) showed a similar transcript profile.
Two GH43 endoarabinases showed different expression profiles, $a b n A$ (NRRL3_00092) was highly expressed in $\triangle \operatorname{lraC}$ and $a b n C$ (NRRL3_05407) was up-regulated in $\Delta \operatorname{lraA}, \Delta \operatorname{lraB}$ and $\triangle \operatorname{lraC}$ compared to the reference.

\section{Discussion}

In this study, we constructed single deletion mutants to confirm in vivo the function of the first three genes of the L-rhamnose catabolic pathway in A. niger ( $\triangle \operatorname{lraA}$, $\triangle \operatorname{lra} B$ and $\Delta \operatorname{lraC})$. These strains, together with the $\triangle$ rhaR mutant, were also used to investigate Lrhamnose-induced transcriptional up-regulation by RhaR in $A$. niger to elucidate the inducer molecule. Comparative growth analysis of the $3 \mathrm{~L}$-rhamnose catabolic mutants showed that individual deletion of $\operatorname{lraA}$, $\operatorname{lraB}$ and $\operatorname{lraC}$ results in an inability to use L-rhamnose as a sole carbon source. This indicates that there are no other enzymes capable of replacing the function of LraA, LraB and LraC, at least at a level that can rescue the growth phenotype on L-rhamnose. These results are in line with a previous study in which a double deletion mutant of $\operatorname{lraA}$ and $\operatorname{lraC}$ in $A$. niger was unable to grow on L-rhamnose [17, 27].

The second aim of our study was to search for $\operatorname{lraD}$ candidate genes in the $A$. niger genome and identify this function by gene deletion. L-rhamnose catabolism genes (RHA1, LRA2, LRA3 and LRA4) have been previously found in a chromosomal gene cluster in S. stipitis $[8,13]$. In $A$. niger, the orthologs of the $S$. stipitis RHA1, LRA2

Table 1 Pectinolytic genes that were significantly down-regulated in the A. niger $\Delta \operatorname{lraA}, \Delta \operatorname{raB}, \Delta \operatorname{lraC}$ and $\Delta$ rhaR strains compared to the reference in L-rhamnose

\begin{tabular}{|c|c|c|c|c|c|c|c|c|c|c|c|c|c|c|c|c|c|}
\hline \multirow[b]{2}{*}{$\underline{\text { Structure }}$} & \multirow{2}{*}{\multicolumn{2}{|c|}{ Enzyme Gene }} & \multirow[b]{2}{*}{ A. niger NRRL3 } & \multirow[b]{2}{*}{ Mean Ref } & \multirow[b]{2}{*}{ Mean $\triangle$ IraA } & \multirow[b]{2}{*}{ Mean $\Delta / r a B$} & \multirow[b]{2}{*}{ Mean $\Delta / r a C$} & \multirow[b]{2}{*}{ Mean $\Delta r h a R$} & \multirow[b]{2}{*}{$\mathrm{ref} / \Delta / \mathrm{raA}$} & \multicolumn{6}{|c|}{$\begin{array}{l}\text { RNA-sequencing } \\
\text { Fold change }\end{array}$} & \multirow{2}{*}{\multicolumn{2}{|c|}{ 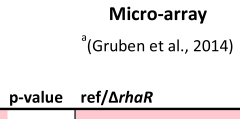 }} \\
\hline & & & & & & & & & & p-value & ref/ $\Delta / r a B$ & p-value & ref/ $\Delta / r a C$ & $p$-value $r$ & ref/ArhaR & & \\
\hline HGA & PAE & paeA & NRRL3_06053 & 26.255 & 29.195 & 20.51 & 22.25 & 8.8 & 0.899298 & 0.58654 & $\quad 1.28011$ & 0.3351 & 1.18 & 0.518 & 2.9835227 & $\begin{array}{l}7 \\
\end{array}$ & 2.6 \\
\hline \multirow[t]{11}{*}{ RG-I } & RGX & $\operatorname{rgxA}$ & NRRL3_02832 & 127.42 & 3.67 & 8.68 & 2.865 & 2.2 & 34.71935 & 0.05061 & 14.6797 & 0.0549 & 44.47469 & 0.0501 & 57.918182 & $2 \quad 0.0496$ & 43.2 \\
\hline & & $\mathrm{rgxB}$ & NRRL3_08631 & 63.325 & 0.79 & 2.8 & 0.1 & 0 & 80.15823 & 0.0084 & 22.6161 & 0.0092 & 633.25 & $50.0082 \ln$ & & 0.0082 & 139.3 \\
\hline & & $\mathrm{rgxC}$ & NRRL3_10559 & 5.865 & 0.37 & 0.75 & 0.37 & 0.475 & 15.85135 & 0.00052 & 7.82 & 0.002 & 15.85135 & 0.0017 & 12.347368 & $\begin{array}{ll}3 & 0.0082\end{array}$ & 20.6 \\
\hline & RHA & & NRRL3_02162 & 2557.12 & 109.145 & 219.98 & 56.52 & 36.89 & 23.42865 & 0.0343 & $\quad 11.6243$ & 0.0375 & 45.24275 & 0.0329 & 69.31743 & $\begin{array}{ll}3 & 0.0325\end{array}$ & 120.9 \\
\hline & & & NRRL3_03279 & 432.225 & 80.685 & 111.91 & 4.92 & 4.85 & 5.356944 & 7. 0.01862 & 3.86226 & 0.0248 & 87.85061 & 0.0125 & 89.118557 & $7 \quad 0.0125$ & 149.5 \\
\hline & & & NRRL3_04245 & 38.465 & 0.295 & 0.51 & 0.295 & 0.305 & 130.3898 & 3 0.05899 & 75.4216 & 0.0596 & 130.3898 & 0.059 & 126.11475 & 0.059 & 15.1 \\
\hline & & & NRRL3_07520 & 330.96 & 33.23 & 71.24 & 13.2 & 14.14 & 9.959675 & $5 \quad 0.01207$ & 4.6457 & 0.0194 & 25.07273 & $3 \quad 0.0106$ & 23.405941 & $1 \quad 0.0107$ & 47.8 \\
\hline & URH & urhgA & NRRL3_00839 & 1298.4 & 105.04 & 150.43 & 21.435 & 21.485 & 12.36096 & 5 0.01101 & 8.63122 & 0.012 & 60.57359 & 0.0096 & 60.432627 & $7 \quad 0.0096$ & 48.3 \\
\hline & RGL & $\mathrm{rg} \mid \mathrm{B}$ & NRRL3_10115 & 2076.07 & 53.1 & 140.88 & 7.925 & 10.52 & 39.09736 & 0.01548 & 14.7364 & 0.0172 & 261.9647 & 0.0148 & 197.34506 & $5 \quad 0.0149$ & 168.5 \\
\hline & RGAE & rgaeA & NRRL3_00169 & 193.745 & 52.44 & 49.795 & 16.005 & 12.555 & 3.694603 & 3 0.05302 & 3.89085 & 0.0523 & 12.10528 & 30.0324 & 15.431701 & $1 \quad 0.0312$ & 18.6 \\
\hline & & rgaeB & NRRL3_07501 & 329.7 & 62.09 & 70.26 & 21.185 & 17.705 & 5.310034 & $\begin{array}{r}+\quad 0.03178 \\
\end{array}$ & 4.69257 & 0.0355 & 15.5629 & 0.0242 & 18.621858 & $\begin{array}{|ll|}8 & 0.0237 \\
\end{array}$ & 27.7 \\
\hline \multirow[t]{2}{*}{ sc } & LAC & lacc & NRRL3_11738 & 130.41 & 9.745 & 20.22 & 3.54 & 7.09 & 13.38225 & 0.00075 & 6.44955 & 0.0016 & 36.83898 & 30.0007 & 18.393512 & $2 \quad 0.0007$ & 25.4 \\
\hline & FAE & faeB & NRRL3_02931 & 30.005 & 9.73 & 8.03 & 8.55 & 7.605 & 3.083762 & $2 \quad 0.14571$ & 3.73661 & 0.1297 & 3.509357 & 0.1372 & 3.9454306 & $\begin{array}{r}6 \quad 0.1206 \\
\end{array}$ & 3 \\
\hline
\end{tabular}

Based on Gruben, B.S., M. Zhou, A. Wiebenga, J. Ballering, K.M. Overkamp, P.J. Punt \& R.P. de Vries, (2014) Aspergillus niger RhaR, a regulator involved in L-rhamnose release and catabolism. Appl Microbiol Biotechnol 98: 5531-5540

The expression levels are mean values of duplicate samples. Genes with values higher than 120 are considered highly expressed and marked red.

Genes with values lower than 20 are considered low expressed and marked green.

The fold change is the difference in expression between the reference strain and the deletion mutants.

The cutoff for differential expression is fold change $>1.5$ (cells marked red if upregulated and green if downregulated) and $p$-value $<0.05$ (cells marked yellow). HGA homogalacturonan, RG-I Rhamnogalacturonan-I, SC side chains, PAE = pectin acetyl esterase, RGX = exorhamnogalacturonase

$\mathrm{RHA}$ = endorhamnogalacturonase, $\mathrm{URH}=$ unsaturated rhamnogalacturonyl hydrolase, $\mathrm{RGL}$ = rhamnogalacturonan lyase

$\mathrm{RGAE}=$ rhamnogalacturonan acetyl esterase, $\mathrm{LAC}=$ beta-galactosidase, $\mathrm{FAE}=$ feruloyl esterase. 
and LRA3 genes, the $\operatorname{lraA}$, $\operatorname{lraB}$ and $\operatorname{lraC}$ genes, are clustered with rhaR on chromosome II, but the cluster does not contain an LRA4 homolog (lraD) [15]. In this study we selected three candidate genes for $\operatorname{lraD}$ that were specifically up-regulated in L-rhamnose and which all have similar PFAM and InterPro domains to those found in LRA4 of S. stipitis. However, deletion of these genes did not reduce growth on L-rhamnose, suggesting that neither of them encodes an L-KDR specific aldolase with a key role in L-rhamnose metabolism. Because the five remaining genes in the $A$. niger genome with a dihydrodipicolinate synthetase family domain (PF00701) were not induced on L-rhamnose or not expressed in any condition (Additional file 4: Table S4), it is very unlikely that these genes are involved in L-rhamnose metabolism in A. niger. A possibility is that the real lraD gene of $A$. niger belongs to a different aldolase family than the LRA4 of S. stipitis. Also, we cannot exclude that an alternative enzyme could convert L-KDR in the L-rhamnose pathway of $A$. niger. In Sphingomonas sp., a gene cluster consisting of LRA1-3, LRA5 and LRA6 has been found. LRA5 and LRA6 were assigned as new enzymes, L-KDR-4-dehydrogenase (KDRDH) and 2, 4-diketo-3-deoxy-L-rhamnonate hydrolase (DDRH), respectively [11]. LRA5 (KDRDH) was identified as an NAD+ specific enzyme belonging to the short-chain dehydrogenase/reductase (SDR) superfamily [11] and has been shown to convert L-KDR to 2, 4-diketo3-deoxy-L-rhamnonate. Interestingly, another KDRDH belonging to the medium chain dehydrogenase reductase (MDR) superfamily has been biochemically characterized in Sulfobacillus thermosulfidooxidans and was found to catalyze the same metabolic reaction [10]. We analysed and compared the PFAM domains in the protein sequences of LRA5 of both species. The KDRDH from Sphingomonas $s p$. contains an enoyl-(Acyl carrier protein) reductase domain (PF13561) and the KDRDH from the acidophile $S$. thermosulfidooxidans contains an alcohol dehydrogenase GroES-like domain (PF08240) and a zincbinding dehydrogenase domain (PF00107). In A. niger, a putative alcohol dehydrogenase (NRRL3_01492) is present in the lraA-C cluster (Fig. 1c), which is 33-fold upregulated in the micro-array data of $A$. niger wild-type in L-rhamnose compared to D-glucose [15]. This putative alcohol dehydrogenase contained the same PFAM domains as KDRDH from $S$. thermosulfidooxidans. Interestingly, this putative alcohol dehydrogenase is well conserved within the genomes of the other Aspergilli [15]. This putative alcohol dehydrogenase might be a likely candidate in A. niger, to convert L-KDR into 2, 4-diketo-3deoxy-L-rhamnonate.

The third aim was to study the transcript profiles of the L-rhamnose-induced genes in the metabolic deletion mutants to identify the inducer of RhaR in A. niger. In our RNA-seq analysis, genes involved in the L-rhamnose catabolism and in RG-I degradation were significantly lower expressed in $\triangle l r a A, \Delta l r a B$ and $\Delta l r a C$ mutants compared to the reference strain. Our results revealed that 12 of the 23 RG-I related pectinolytic genes were $>1.5$ fold down-regulated in all the deletion strains compared to the reference strain on L-rhamnose. In the D-galacturonic acid pathway in $A$. niger, deletion of $g a a A$ and $g a a B$ resulted in reduced expression profiles of pectinolytic genes on D-galacturonic-acid compared to the reference [21], while deletion of $\mathrm{gaaC}$ resulted in up-regulation of these genes whereas no difference was observed for the deletion of gaaD. This study also demonstrated that the inducer of the galacturonic acid degradation route is 2-keto-3-deoxy-L-galactonate, which is the substrate for $\mathrm{GaaC}$, indicating that accumulation of an intermediate due to deletion of a pathway gene allows the identification of the inducer of the pathway. Since the expression of L-rhamnose-responsive genes was reduced in the strains in which $\operatorname{lraA}, \operatorname{lraB}$ or lraC were deleted, this indicates that none of these deletions results in accumulation of the inducer of the pathway regulator (RhaR). The expression did not reduce to zero for all the known rhamnose catabolic genes in all the strains, suggesting that there is either a basal non-regulated expression of these genes or alternatively that they are also under control of other regulatory systems. Based on an alignment between TRC1 in $S$. stipitis and RhaR in A. niger, regions of the DNA Binding domain are conserved. This correlates with the phylogenetic analysis performed in Koivistoinen et al.,2012. Therefor RhaR appears to be an orthologue of TRC1. Also, since the transcription factor RhaR is conserved within the genomes of the other Aspergilli, and is also conserved in fungal species, we postulate that the product of the $\mathrm{LraC}$ reaction, $\mathrm{L}-\mathrm{KDR}$, is the inducer of the system. In S. stipitis another transcription factor than TRC1 has been found in the L-rhamnose cluster, FST14. This transcription factor was more up-regulated than TRC1 on L-rhamnose and it might be co-regulating the pectinolytic genes together with TRC1 [13]. This could also be the case in $A$. niger as previously suggested [15], even though we could not find an orthologue for this regulator in $A$. niger.

The L-rhamnose transporter encoding gene and the metabolic genes are L-rhamnose-induced and in the qPCR and RNA-seq analysis they have a similar gene expression profile in the metabolic and rhaR deletion mutants. These results and those of Sloothaak et al., 2016 [6] indicate that L-rhamnose is predominantly transported via the RhtA transporter and then converted through the L-rhamnose metabolic pathway.

Previously it was shown that only a small concentration of L-rhamnose is enough to induce the system [6], as also observed for the $\mathrm{D}$-xylose regulatory system in $A$. 
niger [30]. The very low expression levels of $\operatorname{lra} A, \operatorname{lraB}$, rhaR, $\operatorname{rglB}, \operatorname{rgx} A$, rgaeA obtained in $\triangle \mathrm{raC}$ in the presence of L-rhamnonate showed that the third step is indeed necessary to generate the inducer. In the $\triangle \operatorname{lraA}$ and $\triangle \mathrm{lraB}$ strains the inability to generate the inducer from L-rhamnose can be overcome by supplying L-rhamnonate instead, which is the product of LraB. This then also explains why $\Delta \operatorname{lra} A$ and $\Delta \operatorname{lraB}$ can still grow on L-rhamnonate, while $\Delta l r a C$ cannot. RNA-seq combined with the qPCR results of the L-rhamnose metabolic mutants demonstrated that L-rhamnose, L-rhamnono- $\gamma$-lactone and L-rhamnonate are not the inducers of RhaR. Interestingly and in contrast to transfer to L-rhamnose, upon transfer to L-rhamnonate the reference, $\triangle \operatorname{lraA}$ and $\triangle \mathrm{lraB}$ strains showed similar expression levels in all the genes tested. L-rhamnonate is unlikely to use the L-rhamnose transporter RhtA, due to its different chemical nature. The higher levels of expression observed in the reference, $\triangle \mathrm{lraA}$ and $\Delta \mathrm{lraB}$ strains suggest that by using L-rhamnonate as carbon source which avoids the first 2 steps in the L-rhamnose metabolic pathway, its metabolism still leads to induction of the L-rhamnose pathway. But induction requires a functional LraC since induction is severely affected in the $\operatorname{lraC}$ deletion strain.

Because the gene or genes involved in the conversion of L-KDR remain unknown in A. niger, studying metabolic gene deletion mutants further downstream of the LraC step is required to unambiguously establish the identity of the inducer. At this point in time we do not know whether L-KDR is converted by a yet unknown aldolase into L-lactaldehyde and pyruvate or, alternatively by LRA 5 and LRA6 homologs to L-lactate and pyruvate. The putative alcohol dehydrogenase present in the cluster, which on the basis of the PFAM domains found and its rhamnose-responsive expression, makes it more likely that the L-rhamnonate degradation pathway involves formation of 2, 4-diketo-3-deoxy-L-rhamnonate from L-KDR. The final C3 metabolites formed (pyruvate and L-lactaldehyde or L-lactate) via these two nonphoshorylating pathways have to be further metabolized via gluconeogenesis. These metabolites are already part of central metabolism and are not expected to play any role in the induction of the rhamnose pathway itself. Lactaldehyde dehydrogenase is involved in the conversion of L-lactaldehyde to L-lactate. Several putative lactaldehyde dehydrogenases (AldA) based on a characterized AldA in E.coli were found in A. niger [31]. The first putative gene (NRRL3_11302) shares 33.3\% identity with AldA from $E$. coli and it is significantly up-regulated in the reference strain compared to all the deletion mutants. Also, one putative lactate dehydrogenase (LDH; NRRL3_07593) that catalyzes the reaction from lactate to pyruvate, shares $37.4 \%$ identity with a characterized LDH from Rhizopus oryzae [32]. RNA-seq results showed that this putative gene is not induced after $2 \mathrm{~h}$ of transfer. This potentially leaves us with two options for the identity of the inducer, viz. L-KDR or 2, 4-diketo-3-deoxy-L-rhamnonate, and further work will be required to discriminate between these two options.

\section{Conclusion}

In conclusion, this study confirmed the function of $\operatorname{lra} A, \operatorname{lra} B$ and $\operatorname{lra} C$ in the $A$. niger L-rhamnose catabolic pathway in vivo. Our search for candidate genes for the L-KDR aldolase ( $r a D)$ resulted in selection and deletion of three genes, but none of them showed to have a role in the L-rhamnose pathway. Based on further conserved domain searches with previously characterized L-rhamnose metabolic genes, we suggest that L-KDR may be converted via an unknown aldolase or via an unknown KDRDH.

Gene expression profiling in the wild-type and the L-rhamnose metabolic mutants demonstrated that Lrhamnose, L-rhamnono- $\gamma$-lactone and L-rhamnonate are not the inducers of the RhaR-regulated genes. The very low expression levels of the first two metabolic genes ( $\operatorname{lraA}$ and $\operatorname{lraB}$ ), three RGI-specific genes ( $r g l B, r g x A, r g a e A)$ and rhaR observed in $\triangle l r a C$ in the presence of L-rhamnonate showed that the third step is necessary to generate an inducer in order to activate these genes. In summary, these results thus strongly suggest that the L-rhamnose pathway intermediate L-KDR is the real inducer of RhaR-regulated genes in A. niger.

\section{Additional files}

Additional file 1: Table S1. Strains used in this study. (PDF $104 \mathrm{~kb}$ )

Additional file 2: Table S2. Primers used in this study. Overlapping sequences for fusion PCR are written in bold. (PDF $80 \mathrm{~kb}$ )

Additional file 3: Table S3. Primers used in this study to generate the gene fragments for qRT-PCR analysis. (PDF $252 \mathrm{~kb}$ )

Additional file 4: Table S4. Identification of candidate LraD genes in Aspergillus niger. The data are organized according to the fold changes (highest to smallest). The fold change is the difference in expression between the wild-type on rhamnose over glucose. $p$-value $<0.05$ (cells marked yellow). In red are indicated the three $\mathrm{LraD}$ candidates selected. (XLSX $17 \mathrm{~kb}$ )

Additional file 5: Table S5. Putative transporter genes in A. niger and their expression levels in the $\Delta / r a A, \Delta / r a B, \Delta / r a C, \Delta r h a R$ mutants and the reference strain after a $2 \mathrm{~h}$ transfer to $\mathrm{L}$-rhamnose. The expression levels are mean values of duplicate samples. Genes with values higher than 120 FPKM are considered highly expressed and marked red. Genes with values lower than 20 are considered low expressed and marked green. The fold change is the difference in expression between the reference strain and the deletion mutants. The cut-off for differential expression is fold change $>1.5$ (cells marked red if up-regulated and green if downregulated) and $p$-value $<0.05$ (cells marked yellow). (XLSX $524 \mathrm{~kb}$ )

Additional file 6: Table S6. Genes of $A$. niger encoding pectinolytic enzymes and their expression in $\Delta / r a A, \Delta / r a B, \Delta / r a C, \Delta r h a R$ mutants and the reference strain after a $2 \mathrm{~h}$ transfer to $\mathrm{L}$-rhamnose. The expression 
levels are mean values of duplicate samples. Genes with values higher than 120 are considered highly expressed and marked red. Genes with values lower than 20 are considered low expressed and marked green. The fold change is the difference in expression between the reference strain and the deletion mutants. The cutoff for differential expression is fold change $>1.5$ (cells marked red if upregulated and green if downregulated) and $p$-value $<0.05$ (cells marked yellow). $\mathrm{HGA}=$ homogalacturonic acid, $\mathrm{XGA}=$ xylogalacturonan, RG-I = Rhamnogalacturonan-I, SC = side chains. (XLSX $624 \mathrm{~kb})$

\section{Abbreviations}

CM: complete medium; GEO: Gene Expression Omnibus; HGA: Homogalacturonan; L-KDR: 2-keto-3-deoxy-L-rhamnonate; MM: Minimal medium; RG-I: Rhamnogalacturonan I; RG-II: Rhamnogalacturonan II; RhaR: Lrhamnose responsive transcription factor; RhtA: L-rhamnose transporter; XGA: Xylogalacturonan

\section{Acknowledgements}

We thank Joanna Kowalczyk for providing the A. niger reference strain.

\section{Funding}

CK and MVA-P were supported by a grant of the Dutch Technology Foundation STW, Applied Science division of NWO, and the Technology Program of the Ministry of Economic Affairs 016.130 .609 to RPdV.

\section{Availability of data and materials}

The data sets supporting the results of this article are available in the GEO repository with accession number: GSE99865.

\section{Authors' contributions}

RPdV conceived the study. RPdV and EB designed experiments. CK and RK performed the experiments. MVAP performed the bioinformatics analysis. CK analysed the data. CK, EB, JV and RPdV interpreted the data and wrote the manuscript. All authors read and approved the final manuscript.

\section{Ethics approval and consent to participate}

Not applicable.

\section{Consent for publication}

Not applicable.

\section{Competing interests}

All authors declare that they have no competing interests.

\section{Publisher's Note}

Springer Nature remains neutral with regard to jurisdictional claims in published maps and institutional affiliations.

Received: 8 June 2017 Accepted: 18 October 2017

Published online: 06 November 2017

\section{References}

1. Voragen AGJ, Coenen G, Verhoef RP, Schols HA. Pectin, a versatile polysaccharide present in plant cell walls. Struct Chem. 2009;20:263-75.

2. Ha MA, Vietor RJ, Jardine GD, Apperley DC, Jarvis MC. Conformation and mobility of the arabinan and galactan side-chains of pectin. Phytochemistry. 2005;66:1817-24.

3. Lau JM, McNeil M, Darvill AG, Albersheim P. Structure of the backbone of rhamnogalacturonan I, a pectic polysaccharide in the primary cell walls of plants. Carbohydr Res. 1985;137:111-25.

4. de Vries RP, Kester HCM, Poulsen CH, Benen JAE, Visser J. Synergy between accessory enzymes from Aspergillus in the degradation of plant cell wall polysaccharides. Carbohydr Res. 2000;327:401-10

5. de Vries RP, Visser J: Aspergillus enzymes involved in degradation of plant cell wall polysaccharides. Microbiol Mol Biol Rev 2001, 65:497-522.

6. Sloothaak J, Odoni DI, dos Santos VAP M, Schaap PJ, Tamayo-Ramos JA. Identification of a novel L-rhamnose uptake transporter in the filamentous fungus Aspergillus niger. PLoS Genet. 2016;12:e1006468.
7. Takagi Y, Sawada H. The metabolism of L-rhamnose in Escherichia coli. Biochim Biophys Acta (BBA) - Specialized Sect Enzymological Sub. 1964;92:10-7

8. Watanabe S, Saimura M, Makino K. Eukaryotic and bacterial gene clusters related to an alternative pathway of nonphosphorylated L-rhamnose metabolism. J Biol Chem. 2008;283:20372-82.

9. Twerdochlib AL, Pedrosa FO, Funayama S, Rigo LU. L-rhamnose metabolism in Pichia stipitis and Debaryomyces polymorpus. Can J Microbiol. 1994;40:896-902.

10. Bae J, Kim SM, Lee SB. Identification and characterization of 2-keto-3deoxy-L-rhamnonate dehydrogenase belonging to the MDR superfamily from the thermoacidophilic bacterium Sulfobacillus thermosulfidooxidans: implications to L-rhamnose metabolism in archaea. Extremophiles. 2015. 19:469-78.

11. Watanabe S, Makino K. Novel modified version of nonphosphorylated sugar metabolism-an alternative L-rhamnose pathway of Sphingomonas sp. FEBS J. 2009:276:1554-67.

12. un Rigo L, Nakano M, Veiga LA, Feingold DS. L-rhamnose dehydrogenase of Pullularia pullulans. Biochim Biophys Acta (BBA) - Enzymol. 1976:445:286-93.

13. Koivistoinen $O M$, Arvas $M$, Headman JR, Andberg $M$, Penttilä $M$, Jeffries TW, Richard P. Characterisation of the gene cluster for L-rhamnose catabolism in the yeast Scheffersomyces (Pichia) stipitis. Gene. 2012;492:177-85.

14. Koivistoinen $\mathrm{OM}$, Hilditch $\mathrm{S}$, Voutilainen $\mathrm{SP}$, Boer H, Penttilä M, Richard P. Identification in the yeast Pichia stipitis of the first L-rhamnose-1dehydrogenase gene. FEBS J. 2008;275:2482-8.

15. Gruben BS, Zhou M, Wiebenga A, Ballering J, Overkamp KM, Punt PJ, de Vries RP. Aspergillus niger RhaR, a regulator involved in L-rhamnose release and catabolism. Appl Microbiol Biotechnol. 2014;98:5531-40.

16. Motter FA, Kuivanen J, Keränen $H$, Hilditch $S$, Penttilä M, Richard P. Categorisation of sugar acid dehydratases in Aspergillus niger. Fungal Genet and Biol. 2014;64:67-72.

17. Kuivanen J, Richard P. Engineering a filamentous fungus for L-rhamnose extraction. AMB Express. 2016;6:27.

18. Pardo $\mathrm{E}_{3}$ Orejas $\mathrm{M}$. The Aspergillus nidulans Zn (II)2Cys6 transcription factor AN5673/RhaR mediates L-rhamnose utilization and the production of $a-L-$ rhamnosidases. Microb Cell Factories. 2014:13:161.

19. Battaglia E, Hansen SF, Leendertse A, Madrid S, Mulder H, Nikolaev I, de Vries RP. Regulation of pentose utilisation by AraR, but not XInR, differs in Aspergillus nidulans and Aspergillus niger. Appl Microbiol Biotechnol. 2011:91:387-97.

20. Hasper AA, Visser J, de Graaff LH: The Aspergillus niger transcriptional activator XInR, which is involved in the degradation of the polysaccharides xylan and cellulose, also regulates D-xylose reductase gene expression. Mol Microbiol 2000, 36:193-200.

21. Alazi E, Khosravi C, Homan TG, du Pré S, Arentshorst M, Di Falco M, Pham TTM, Peng M, Aguilar-Pontes MV, Visser J, et al. 2-Keto-3-deoxy-Lgalactonate mediates the induction of genes involved in D-galacturonic acid utilization in Aspergillus niger. FEBS Lett.

22. Alazi E, Niu J, Kowalczyk JE, Peng M, Aguilar Pontes MV, van Kan JA, Visser J, de Vries RP, Ram AF. The transcriptional activator GaaR of Aspergillus niger is required for release and utilization of D-galacturonic acid from pectin. FEBS Lett. 2016;590:1804-15.

23. de Vries RP, Burgers K, van de Vondervoort PJI, Frisvad JC, Samson RA, Visser J: A new black Aspergillus species, A. vadensis, is a promising host for homologous and heterologous protein production. Appl Environ Microbiol 2004, 70:3954-3959.

24. Kusters-van Someren M, Flipphi M, de Graaff $L H$, van den Broeck $H$, Kester $H$, Hinnen A, Visser J. Characterisation of the Aspergillus niger pelB gene: structure and regulation of expression. Mol Gen Genet. 1992;234:113-20.

25. Li H, Durbin R. Fast and accurate long-read alignment with burrowswheeler transform. Bioinformatics. 2010;26:589-95.

26. Langmead B, Trapnell C, Pop M, Salzberg SL. Ultrafast and memory-efficient alignment of short DNA sequences to the human genome. Genome Biol. 2009;10:R25

27. Li B, Dewey CN. RSEM: accurate transcript quantification from RNA-Seq data with or without a reference genome. BMC Bioinformatics. 2011:12:323.

28. Edgar R, Domrachev M, Lash AE. Gene expression omnibus: NCBI gene expression and hybridization array data repository. Nucleic Acids Res. 2002;30:207-10

29. Livak KJ, Schmittgen TD. Analysis of relative gene expression data using real-time quantitative PCR and the $2^{-\Delta \Delta C}$ T method. Methods. 2001;25:402-8. 
30. van der Veen D, Oliveira JM, van den Berg WA, de Graaff LH. Analysis of variance components reveals the contribution of sample processing to transcript variation. Appl Environ Microbiol. 2009;75:2414-22.

31. Di Costanzo L, Gomez GA, Christianson DW: Crystal structure of lactaldehyde dehydrogenase from Escherichia coli and inferences regarding substrate and cofactor specificity. J Mol Biol 2007, 366:481-493.

32. Skory CD. Isolation and expression of lactate dehydrogenase genes from Rhizopus oryzae. Appl Environ Microbiol. 2000;66:2343-8.

Submit your next manuscript to BioMed Central and we will help you at every step:

- We accept pre-submission inquiries

- Our selector tool helps you to find the most relevant journal

- We provide round the clock customer support

- Convenient online submission

- Thorough peer review

- Inclusion in PubMed and all major indexing services

- Maximum visibility for your research

Submit your manuscript at www.biomedcentral.com/submit 